\title{
New Results on Minimax Regret Single Facility Ordered Median Location Problems on Networks ${ }^{\star}$
}

\author{
Justo Puerto, Antonio M. Rodriguez-Chia, and Arie Tamir \\ ${ }^{1}$ Facultad de Matemáticas. Universidad de Sevilla \\ ${ }^{2}$ Facultad de Ciencias. Universidad de Cádiz \\ ${ }^{3}$ School of Mathematical Sciences. Tel Aviv University
}

\begin{abstract}
We consider the single facility ordered median location problem with uncertainty in the parameters (weights) defining the objective function. We study two cases. In the first case the uncertain weights belong to a region with a finite number of extreme points, and in the second case they must also satisfy some order constraints and belong to some box, (convex case). To deal with the uncertainty we apply the minimax regret approach, providing strongly polynomial time algorithms to solve these problems.
\end{abstract}

Keywords: Analysis of algorithms, networks, facility location.

\section{Introduction}

The definition of an instance of an optimization problem requires the specification of the problem parameters, like resource limitations and coefficients of the objective function in a linear program, or edge capacities in network flow problems, which may be uncertain or imprecise. Uncertainty/imprecision can be structured through the concept of a scenario which corresponds to an assignment of plausible values to the model parameters. In general, the set of all admissible scenarios may depend on the properties of the underlying model, and on some possible known relationships between the model parameters. Nevertheless, we note that in most published studies it has been assumed that each parameter can independently take on values in some prespecified interval. This is the so called interval data approach.

One of the most common approaches to deal with uncertain data is throughout the minimax absolute regret criterion. In this approach the goal is to minimize the worst case opportunity loss, defined as the difference between the achieved objective-function value and the optimal objective-function value under the realized scenario. We refer the reader to $22315|8| 12] 17$, where recent results on this subject for general optimization problems are described.

\footnotetext{
* Partially supported by grants n. MTM2004-0909, SAB2005-0095, P06-BFM-01366, MTM2007-67433-C02. 
We were motivated by facility location optimization problems where the exact nature of the optimality criteria was uncertain. Consider, for example, a location model in which the central administration subsidizes the transportation cost of the users only after the establishment of a server. Hence, at the moment when the facility has to be established, it is not yet clear what is the cost function that the central administration will apply for determining the magnitude of the subsidy. The administration may decide that the subsidy will be proportional to the distances traveled to the facility by all the customers with the exception of some unknown subset of outliers, e.g., those customers who are too close or too far to the server. The uncertainty is in the size or the exact definition of the set of outliers. In such a case, the criterion to be chosen 'a priori' for the location of the server might be taken as minimizing the regret of the decision among a certain family of criteria.

The approach that we suggest to deal with this uncertainty is to limit ourselves to certain families of objective functions, and apply the approach of minimizing the maximum regret with respect to the selection of an objective within the prespecified. Our approach is different from the approach in the existing literature on minimax regret facility location models, where the objective function used is usually assumed to be known and certain (445]). Specifically, in this paper, we will restrict ourselves to the family of ordered median functions (OMF) which has been studied extensively in the last decade in location theory, see [915]. This family unifies large variety of criteria used in location modeling.

The OMF is a real function defined on $\mathbb{R}^{n}$ and characterized by a sequence of reals, $\lambda=\left(\lambda_{1}, \ldots, \lambda_{n}\right)$. For a given point $z \in \mathbb{R}^{n}$, let $\bar{z} \in \mathbb{R}^{n}$ be the vector obtained from $z$ by sorting its components in nondecreasing order. In the context of a single facility (server) location model with $n$ demand points, the OMF objective is applied as follows. Let $x$ denote the location of the server in the respective metric space, and let $z(x)$ denote the vector of the $n$ weighted distances of the demand points to the server at $x$. The value of the ordered median objective at $x$ is then defined as the scalar product of $\lambda$ with $\bar{z}(x)$. As noted above, this function unifies and generalizes the classical and most common criteria, i.e., center and median, used in location modeling. (We get the median objective when $\lambda_{i}=1, i=1, \ldots, n$, and the center objective when $\lambda_{i}=0, i=1, \ldots, n-1$ and $\lambda_{n}=1$.) Another important case is the $k$-centrum objective, where the goal is to minimize the sum of the $k$-largest weighted distances to the server. This case is characterized by $\lambda_{i}=0, i=1, \ldots, n-k$, and $\lambda_{i}=1, i=n-k+1, \ldots, n$. In addition to the above examples, the ordered median objective generalizes other popular criteria often used in facility location studies, e.g., centdian and $\left(k_{1}, k_{2}\right)$-trimmed mean.

In this paper, we solve a variety of single facility minimax regret ordered median problems on general networks finding best solutions on each edge. The reader can find further details in [16. A summary of the results is given in Table 1 .

The paper is organized as follows. In Section 2, we present the single facility ordered median problem in general networks. Section 3 introduces the minimax 
Table 1. Summary of results

\begin{tabular}{|l||l|}
\hline \multicolumn{1}{|c|}{ Objective function } & \multicolumn{1}{|c|}{ Complexity } \\
\hline \hline OMF with lower and upper bounds & $O\left(m^{2} n^{4} \log n\right)$ \\
\hline$\left(k_{1}, k_{2}\right)$-trimmed mean & $O\left(m n^{4}\right)$ \\
\hline$k$-centrum & $O\left(m n^{2} \log ^{2} n\right)$ \\
\hline Convex OMF with lower and upper bounds & $O\left(m^{2} n^{6} \log ^{4} n\right)$ \\
\hline Convex OMF with lower and upper bounds on trees & $O\left(n^{6} \log ^{4} n\right)$ \\
\hline
\end{tabular}

regret ordered median problem and some results concerning the convexity of the objective function. In Section 4, we develop strongly polynomial algorithms for this type of problems when the feasible region of the $\lambda$-weights has a finite number of extreme points. Section 5 is devoted to analyze the convex case, which is defined by the property that the $\lambda$-weights are given in nondecreasing order; for this case a strongly polynomial algorithm is developed.

\section{Notation}

Let $G=(V, E)$ be an undirected graph with node set $V=\left\{v_{1}, \ldots, v_{n}\right\}$ and edge set $E,|E|=m$. Each edge $e \in E$, has a positive length $l_{e}$, and is assumed to be rectifiable. In particular, an edge $e$ is identified as an interval of length $l_{e}$, so that we can refer to its interior points. Let $\mathcal{A}(G)$ denote the continuum set of points on the edges of $G$. Each subgraph of $G$ is also viewed as a subset of $\mathcal{A}(G)$, e.g., each edge $e \in E$ is a subset of $\mathcal{A}(G)$. We refer to an interior point on an edge by its distance along the edge to the nodes of the edge.

The edge lengths induce a distance function $d$ on $\mathcal{A}(G)$ and thus $\mathcal{A}(G)$ is a metric space, see [18. We consider a set of nonnegative weights $\left\{w_{1}, \ldots, w_{n}\right\}$, called $w$-weights, where $w_{i}, i=1, \ldots, n$, is associated with node $v_{i}$ and represents the intensity of the demand at this node.

For any $x \in \mathcal{A}(G)$, let $\sigma=\sigma(x)$ be a permutation of the set $\{1, \ldots, n\}$ satisfying $w_{\sigma_{1}} d\left(v_{\sigma_{1}}, x\right) \leq \ldots \leq w_{\sigma_{n}} d\left(v_{\sigma_{n}}, x\right)$. For $i=1, \ldots, n$, denote $d_{(i)}(x)=$ $w_{\sigma_{i}} d\left(v_{\sigma_{i}}, x\right) .\left(d_{(i)}(x)\right.$ is an $i$-th smallest element in the set $\left.\left\{w_{j} d\left(v_{j}, x\right)\right\}_{j}\right)$.

For a given vector $\lambda=\left(\lambda_{1}, \ldots, \lambda_{n}\right)$ with real components, called $\lambda$-weights, the ordered median function on $\mathcal{A}(G)$ is defined as

$$
f_{\lambda}(x):=\sum_{i=1}^{n} \lambda_{i} w_{\sigma_{i}} d\left(v_{\sigma_{i}}, x\right) .
$$

The single facility ordered median problem is to minimize $f_{\lambda}(x)$ over $\mathcal{A}(G)$, 15].

An ordered median function is called convex if $0 \leq \lambda_{1} \leq \ldots \leq \lambda_{n}$.

A point $x \in \mathcal{A}(G)$ is an equilibrium point with respect to a pair of nodes $v_{k}$, $v_{l}, k \neq l$, if $w_{k} d\left(v_{k}, x\right)=w_{l} d\left(v_{l}, x\right)$. A point $x \in \mathcal{A}(G)$ is a bottleneck point if for some $i=1, \ldots, n$, with $w_{i}>0$, and $e \in E, x$ is the unique maximum point of the (concave) function $w_{i} d\left(v_{i}, y\right)$ when $y$ is restricted to be in $e$. Denote by $\mathcal{E} \mathcal{Q}$ the set of all equilibrium and bottleneck points. This set can be a continuum set 
even in the case of a tree network when two nodes are equally weighted. Let $E Q$ be the set consisting of the nodes of $G$ and all the boundary points of $\mathcal{E} \mathcal{Q}$. Let $B$ be the subset consisting of the nodes of $G$ and the bottleneck points in $E Q$. For each $e \in E$ define $E Q(e)=E Q \cap e$ and $B(e)=B \cap e$. Note that $|E Q(e)|=O\left(n^{2}\right)$ and $|B(e)|=O(n)$.

For each $e \in E$ the points in $E Q(e)(B(e))$ induce a partition of the edge $e$ into $|E Q(e)|-1(|B(e)|-1)$ subedges. These subedges (subinterals) are viewed as consecutive subintervals of the rectified edge (interval) $e$.

After computing the distances between all the nodes, the effort to compute and sort the points in $E Q(e)(B(e))$ for each $e \in E$ is $O\left(n^{2}+|E Q(e)| \log n\right)$ $(O(n+|B(e)| \log n))$. Note that for each subinterval in the partition induced by $B(e)$, each function $w_{i} d\left(v_{i}, x\right)$ is linear. Moreover, for each open subinterval in the partition induced by $E Q(e)$, no pair of functions in the collection $\left\{w_{i} d\left(v_{i}, x\right)\right\}_{i}$ intersect.

\section{The Minimax Regret Ordered Median Problem}

We assume that the vector $\lambda$ is unknown and can take on any value in some compact set $\Lambda \subset \mathbb{R}^{n}$. Any $\lambda \in \Lambda$ is called a scenario and represents a possible $\lambda$-weight instance. The minimax regret ordered median optimization problem is formally defined by:

$$
\min _{x \in \mathcal{A}(G)} R(x):=\max _{\lambda \in \Lambda} \max _{y \in \mathcal{A}(G)}\left(f_{\lambda}(x)-f_{\lambda}(y)\right) .
$$

For any choice of the $\lambda$-weights, $E Q$ contains at least one optimal solution for the respective ordered median problem, [15]. Thus, for a given $x \in \mathcal{A}(G)$

$$
\max _{y \in \mathcal{A}(G)}\left(f_{\lambda}(x)-f_{\lambda}(y)\right)=\max _{y \in E Q}\left(f_{\lambda}(x)-f_{\lambda}(y)\right)=f_{\lambda}(x)-f_{\lambda}\left(y^{*}(\lambda)\right),
$$

where $y^{*}(\lambda) \in E Q$ is a minimizer of $f_{\lambda}(u)$ with $u \in \mathcal{A}(G)$. For each fixed $y \in \mathcal{A}(G)$ define

$$
R_{y}(x)=\max _{\lambda \in \Lambda}\left(f_{\lambda}(x)-f_{\lambda}(y)\right) .
$$

By definition, for a given pair $x, y \in A(G)$ the function $f_{\lambda}(x)-f_{\lambda}(y)$ is linear in $\lambda$. Therefore, $R_{y}(x)=\max _{\lambda \in \operatorname{ext}(C H(\Lambda))}\left(f_{\lambda}(x)-f_{\lambda}(y)\right)$, where $C H(\Lambda)$ is the convex hull of $\Lambda$ and $\operatorname{ext}(\mathrm{CH}(\Lambda))$ is the set of extreme points of $\mathrm{CH}(\Lambda)$.

With this notation

$$
R(x)=\max _{y \in E Q} R_{y}(x) .
$$

Consider an edge $e \in E$ and let $x_{1}^{e}<\ldots<x_{q(e)}^{e}$, be the sequence of equilibrium points in $E Q(e) .\left(q(e)=|E Q(e)|\right.$.) Similarly, let $\bar{x}_{1}^{e}<\ldots<\bar{x}_{b(e)}^{e}$, be the sequence of bottleneck points in $B(e)$. $(b(e)=|B(e)|$.) (See Figure 1). From the definition of equilibrium and bottleneck points and the above notation, we clearly have the following results. 
Lemma 1. Consider a subedge $\left[x_{k}^{e}, x_{k+1}^{e}\right], 1 \leq k<q(e)$. For any $\lambda \in \Lambda$, the function $f_{\lambda}(x)$ is linear on the subedge. For any $y \in E Q$ the function $R_{y}(x)$ is continuous and convex on the subedge. Moreover, if the number of extreme points of $C H(\Lambda)$ is finite $R_{y}(x)$ is also piecewise linear on the subedge.

Lemma 2. For any $y \in E Q$, the function $f_{\lambda}(y)$ is linear in $\lambda$. Let $P \subseteq \mathcal{A}(G)$ be a path such that $f_{\lambda}(x)$ is convex on $P$ for any $\lambda \in \Lambda$. Then for any $y \in E Q$ the function $R_{y}(x)$ is convex on $P$. Moreover, the function $R(x)$ is convex on $P$.

To solve the minimax regret ordered median problem on a general network we will find the best local solution on each edge, i.e., we will solve $m$ subproblems. We refer to each local subproblem as a restricted subproblem.

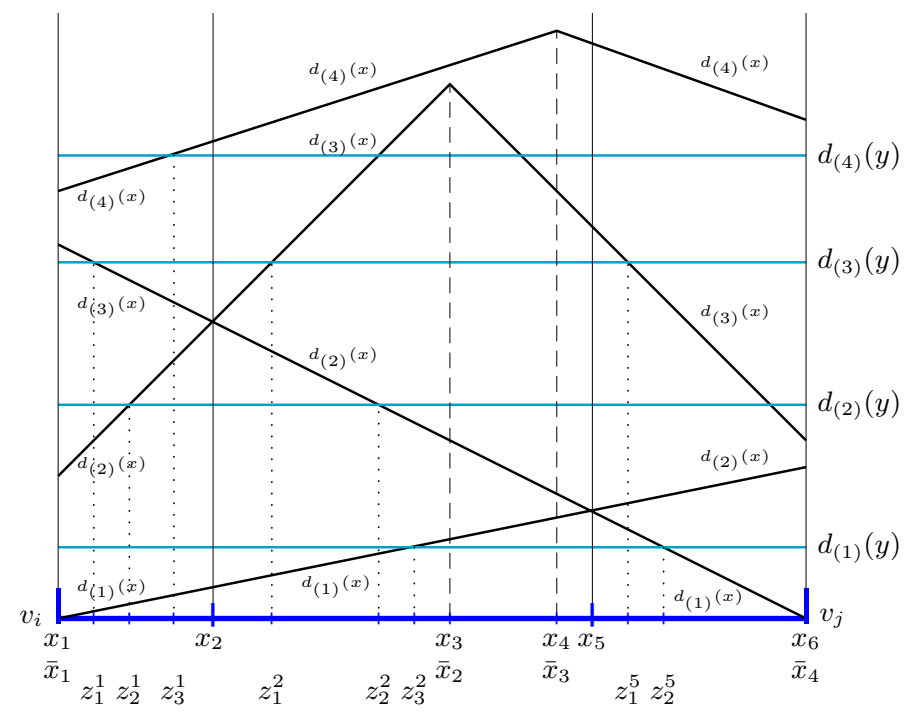

Fig. 1. Equilibrium and bottleneck points

\section{Specific Models}

In this section, we focus on solving restricted subproblems for a variety of sets $\Lambda$, where the number of extreme points of the convex hull of $\Lambda$ is finite. As noted above, we concentrate on finding the best solution on each edge. Hence, we focus on optimizing $R(x)$ on a given edge $e$.

We start with the case where $\Lambda=\left\{\left(\lambda_{1}, \ldots, \lambda_{n}\right): a_{i} \leq \lambda_{i} \leq b_{i}, i=1, \ldots, n\right\}$. The reader may notice that this type of sets is the most common one used in the literature on regret analysis, see [12]. Here, we can strengthen the result in Lemma 1. Consider an edge $e \in E$.

Lemma 3. For each $1 \leq k<q(e)$, and any $y \in E Q$ the function $R_{y}(x)$ is the maximum of $n$ linear functions in $\left[x_{k}^{e}, x_{k+1}^{e}\right]$. 
Lemma 4. For each $1 \leq k<q(e)$, the function $R(x)$ is the upper envelope of $O(n|E Q|)$ linear functions for all $x \in\left[x_{k}^{e}, x_{k+1}^{e}\right]$.

To solve the restricted problem on an edge $e$, we first do some preprocessing on this edge. Assume that we have already computed and sorted the equilibrium points in $E Q(e)$. For each triplet $y \in E Q, v_{i}, v_{j} \in V$, we compute the at most two roots of the equation $w_{i} d\left(x, v_{i}\right)=w_{j} d\left(y, v_{j}\right)$ on $e$. Define $B P(e)$ to be the set consisting of $E Q(e)$ and all these roots. $|B P(e)|=O\left(n^{2}|E Q|\right)$. Moreover, for each $i$ let $z_{i}^{k}(y)$ be the solution, if it exists, to the equation $d_{(i)}(x)=d_{(i)}(y)$ (in the variable $x)$, in the interval $\left[x_{k}^{e}, x_{k+1}^{e}\right]$. Each point $z_{i}^{k}(y)$, is in $B P(e)$.) Finally, we sort the elements in $B P(e)$. The total preprocessing effort is $O\left(n^{2}|E Q| \log n\right)$.

Corollary 1. After spending $O\left(n^{2}|E Q| \log n\right)$ time on preprocessing, for each $1 \leq k<q(e)$ the local minimizer of $R(x)$ over the interval $\left[x_{k}^{e}, x_{k+1}^{e}\right]$ can be computed in $O(n|E Q|)$ time. The optimal solution to the minimax regret ordered median problem on the edge e can be computed in $O(n|E Q \| E Q(e)|)$ time.

The above result implies that for a general network the total time to solve the minimax regret ordered median problem over a box is $O\left(\mathrm{~m}^{2} n^{5}\right)$. The latter bound can be further improved. Focusing on a given edge, we dynamically maintain 11 the upper envelope of $O(|E Q|)$ linear functions which define the function $R(x)$ over a refined subinterval defined by two consecutive elements in the set $B P(e)$. Specifically, following the ordering of the elements in $B P(e)$ we update this envelope. For a given element $u \in B P(e)$, if $u \in E Q(e)$ we may need to update $O(|E Q|)$ linear functions since the ordering or some of the slopes of the functions $\left\{d_{(i)}(x)\right\}$ change. (For each $y \in E Q$ we need to update at most two elements, per pair of indices $j, k$ such that $w_{j} d\left(v_{j}, u\right)=w_{k} d\left(v_{k}, u\right)$, in the sequence $\left\{d_{(i)}(x)-d_{(i)}(y)\right\}_{i}$, or change the slope of a $d_{(i)}(x)$ function, per each $j$ such that $u$ is the maximum of the function $w_{j} d\left(v_{j}, x\right)$ on $e$.) If $u$ coincides with some element $z_{i}^{k}(y)$ defined above, we need to update one function, per each $y \in E Q$ and $j, k$ such that $w_{j} d\left(v_{j}, u\right)=d_{(i)}(u)=d_{(i)}(y)=w_{k} d\left(v_{k}, y\right)$, in the collection. Thus, the total number of insertions and deletions of functions to the collection of $O(|E Q|)$ functions in the upper envelope is $O\left(|E Q| n^{2}\right)$. Using the data structure in Hershberger and Suri 11, each insertion and deletion can be performed in $O(\log n)$ time. Also, the minimum of $R(x)$ over each subinterval connecting two consecutive elements of $B P(e)$ can be computed in $O(\log n)$ time.

Since there are $O\left(n^{2}\right)$ points in $E Q(e)$ and $O\left(n^{2}|E Q|\right)$ points in $B P(e)$ the overall effort to find the best solution on $e$ is $O\left(n^{2}|E Q| \log n\right)$.

Theorem 1. The total time to solve the single facility minimax regret ordered median problem over a box on a general graph is $O\left(m^{2} n^{4} \log n\right)$.

We note that in some important cases the number of extreme points of the convex hull of $\Lambda$ is relatively small. Hence, it may be advantageous to consider these extreme points explicitly. This is the case of the family of $\left(k_{1}, k_{2}\right)$-trimmed mean functions [15, mentioned in the introduction. (Other cases are analyzed in the next section.) For this family $\Lambda=\left\{\left(\lambda_{1}, \ldots, \lambda_{n}\right): \exists k_{1}, k_{2} ; k_{1}+k_{2}<n, \lambda_{1}=\ldots=\right.$ 
$\left.\lambda_{k_{1}}=\lambda_{n-k_{2}+1}=\ldots=\lambda_{n}=0, \lambda_{k_{1}+1}=\ldots=\lambda_{n-k_{2}}=1\right\}$. The extreme points of the convex hull of $\Lambda$ are the vectors of the form $(0, \ldots, 0,1, \ldots, 1,0, \ldots, 0)$. Therefore, in total there are $O\left(n^{2}\right)$ extreme points.

Consider an edge $e \in E$. We claim that for this family, on each interval defined by two consecutive points of $E Q(e)$, the function $R(x)$ can be described as an upper envelope of $O\left(n^{2}\right)$ linear functions. To facilitate the discussion, for each $k=1, \ldots, n$, let $S_{k}(x)=\sum_{i=n-k+1}^{n} d_{(i)}(x)$.

For each $1 \leq s<q(e)$, and for each $x \in\left[x_{s}^{e}, x_{s+1}^{e}\right]$, we have $R(x)=$ $\max _{k_{1}, k_{2} ; k_{1}+k_{2}<n}\left(\left(\sum_{i=k_{1}+1}^{n-k_{2}} d_{(i)}(x)\right)-\varrho_{k_{1}, k_{2}}\right)$, where $\varrho_{k_{1}, k_{2}}=\min _{y \in E Q} \sum_{l=k_{1}+1}^{n-k_{2}} d_{(l)}(y)$. Hence, $R(x)=\max _{k_{1}, k_{2} ; k_{1}+k_{2}<n}\left(S_{n-k_{1}}(x)-S_{k_{2}}(x)-\min _{y \in E Q}\left(S_{n-k_{1}}(y)-S_{k_{2}}(y)\right)\right)$.

Since $R(x)$ is the upper envelope of $O\left(n^{2}\right)$ linear functions, its minimum on the interval $\left[x_{s}^{e}, x_{s+1}^{e}\right]$ can be computed in $O\left(n^{2}\right)$ time using the algorithm in [14. Hence, the solution to the minimax regret ordered median problem on a given edge $e$ for this family of functions, can be obtained in $O\left(n^{2}|E Q(e)|\right)$ time.

Theorem 2. The total time to solve the single facility minimax regret $\left(k_{1}, k_{2}\right)$ trimmed mean problem on a general graph is $O\left(m n^{4}\right)$.

\section{Minimax Regret Convex Ordered Median Problem}

In this section we analyze the minimax regret convex ordered median problem which includes several interesting and most common families of functions used in Location Theory.

The first is the family of $k$-centrum functions where $k$ can vary between 1 and $n$ (see [19]). We have $\Lambda=\left\{\left(\lambda_{1}, \ldots, \lambda_{n}\right): \lambda_{1} \leq \ldots \leq \lambda_{n}\right.$ and $\lambda_{i} \in\{0,1\}, i=$ $1, \ldots, n\}$. The $n$ extreme points of the convex hull of $\Lambda$ are the vectors of the form $(0, \ldots, 0,1, \ldots, 1)$.

Consider an edge $e \in E$. We claim that for this family, on each interval defined by two consecutive points of $B(e)$, the function $R(x)$ can be described as an upper envelope of $n$ convex functions. Indeed, for each $1 \leq s<b(e)$, and for each $x \in\left[\bar{x}_{s}^{e}, \bar{x}_{s+1}^{e}\right], R(x)=\max _{k=1, \ldots, n}\left(\left(\sum_{i=n-k+1}^{n} d_{(i)}(x)\right)-\zeta_{k}\right)$, where $\zeta_{k}=\min _{y \in E Q} \sum_{l=n-k+1}^{n} d_{(l)}(y)$. Hence

$$
R(x)=\max _{k=1, \ldots, n}\left(S_{k}(x)-\min _{y \in E Q} S_{k}(y)\right) .
$$

Note that for each $i=1, \ldots, n$, the function $d\left(v_{i}, x\right)$ is linear over the subinterval $\left[\bar{x}_{s}^{e}, \bar{x}_{s+1}^{e}\right]$. Therefore, for each $k=1, \ldots, n$, the function $\sum_{l=n-k+1}^{n} d_{(l)}(x)$ is convex over the subinterval $\left[\bar{x}_{s}^{e}, \bar{x}_{s+1}^{e}\right]$. (See [15 19]). 
To evaluate $R(x)$ for a given $x$, it is sufficient to sort the elements $\left\{w_{i} d\left(x, v_{i}\right)\right\}$ in order to compute the terms $\left\{S_{k}(x)\right\}_{k}$, and finally find $\max _{k=1, \ldots, n}\left(S_{k}(x)-\right.$ $\left.\min _{y \in E Q} S_{k}(y)\right)$. The minimum of $R(x)$ on the interval $\left[\bar{x}_{s}^{e}, \bar{x}_{s+1}^{e}\right]$ can then be computed in $O\left(n \log ^{2} n\right)$ time by using the parametric approach of Megiddo [13] with the modification in Cole [7. Hence, the solution to the minimax regret ordered median problem on a given edge $e$ for this family of functions can be obtained in $O\left(n \log ^{2} n|B(e)|\right)$ time. (We assume that in the preprocessing phase of the algorithm we have already calculated the terms $\left\{\zeta_{k}\right\}_{k}$. The total effort for this phase is $O\left(m n^{2} \log n\right)$, see [15]).

Theorem 3. The total time to solve the minimax regret $k$-centrum problem on a general graph is $O\left(m n^{2} \log ^{2} n\right)$.

The above analysis and algorithm are also applicable to the more general convex case defined by $\Lambda=\left\{\left(\lambda_{1}, \ldots, \lambda_{n}\right): \lambda_{1} \leq \ldots \leq \lambda_{n}\right.$ and $\left.\lambda_{i} \in[a, b], i=1, \ldots, n\right\}$, where $a$ and $b$ satisfy $0 \leq a \leq b$, see [15. In this case we have

$$
\Lambda=C H\left(\left\{\left(\lambda_{1}, \ldots, \lambda_{n}\right): \lambda_{1} \leq \ldots \leq \lambda_{n} \text { and } \lambda_{i} \in\{a, b\}, i=1, \ldots, n\right\}\right) .
$$

As another example of a convex family of ordered median functions with a small number of extreme points, consider the model corresponding to the $\alpha$ centdian problem, see [15]. In this case, $\Lambda=\left\{\left(\lambda_{1}, \ldots, \lambda_{n}\right): \exists 0 \leq \alpha \leq 1, \lambda_{1}=\right.$ $\ldots=\lambda_{n-1}=\alpha$, and $\left.\lambda_{n}=1\right\}$. We have $\operatorname{ext}(\Lambda)=\{(0, \ldots, 0,1),(1, \ldots, 1)\}$.

\subsection{The Case of Interval Weights and Order Constraints}

In this section, we consider the minimax regret convex ordered median problem where the $\lambda$-weights are in the set,

$$
\Lambda_{\leq}=\left\{\left(\lambda_{1}, \ldots, \lambda_{n}\right): \lambda_{i} \in\left[a_{i}, b_{i}\right] \text { for } i=1, \ldots, n \text {, and } 0 \leq \lambda_{1} \leq \ldots \leq \lambda_{n}\right\} .
$$

Without loss of generality, we may assume that both sequences $\left\{a_{i}\right\}$ and $\left\{b_{i}\right\}$ are nonnegative and nondecreasing. We note that the components of each extreme point of $\Lambda_{\leq}$are elements of the set $A B=\left\{a_{1}, \ldots, a_{n}, b_{1}, \ldots, b_{n}\right\}$. Indeed, let $\lambda$ be an extreme point and suppose without loss of generality that some $\lambda_{i} \notin A B$. Let $1 \leq s \leq i \leq t \leq n$ be such that $\lambda_{s-1}<\lambda_{s}=\lambda_{i}=\lambda_{t}<\lambda_{t+1}$. For $\varepsilon>0$ sufficiently small, consider the vector $\lambda(\varepsilon+)$ defined by setting $\lambda_{j}(\varepsilon+)=\lambda_{j}+\varepsilon$ for $s \leq j \leq t$ and $\lambda_{j}(\varepsilon+)=\lambda_{j}$ otherwise. Similarly, consider the vector $\lambda(\varepsilon-)$ defined by setting $\lambda_{j}(\varepsilon-)=\lambda_{j}-\varepsilon$ for $s \leq j \leq t$ and $\lambda_{j}(\varepsilon-)=\lambda_{j}$ otherwise. The vector $\lambda$ is the midpoint of the interval connecting $\lambda(\varepsilon+)$ and $\lambda(\varepsilon-)$, contradicting the fact that $\lambda$ is an extreme point of $\Lambda_{\leq}$.

Recall that for a given $y \in E Q, R_{y}(x)=\max _{\lambda \in \Lambda_{\leq}}\left(f_{\lambda}(x)-f_{\lambda}(y)\right)$, see (11). Evaluating $R(x)$ for a given $x$ amounts to computing the $|E Q|$ values $R_{y}(x)$ for all $y \in E Q$, see (2).

We propose an algorithm to compute $R_{y}(x)$ for any fixed $y \in E Q$. Consider an edge $e \in E$. Let $\bar{x}_{k}^{e}$ and $\bar{x}_{k+1}^{e}$ be two consecutive elements of $B(e) . f_{\lambda}(x)$ is a 
convex function on $\left[\bar{x}_{k}^{e}, \bar{x}_{k+1}^{e}\right]$, see [15. By Lemma 2, the functions $\left\{R_{y}(x)\right\}_{y \in E Q}$, as well as $R(x)$, are all piecewise linear and convex on $\left[\bar{x}_{k}^{e}, \bar{x}_{k+1}^{e}\right]$.

For a fixed $y \in E Q$ and $x \in\left[\bar{x}_{k}^{e}, \bar{x}_{k+1}^{e}\right]$ the evaluation of $R_{y}(x)$ can be done by solving the following linear program:

$$
\begin{aligned}
R_{y}(x)=\max & c^{T}(x) \lambda-h^{T}(y) \lambda \\
\text { s.t. } & \lambda_{i}-\lambda_{i+1} \leq 0, \quad \forall i=1, \ldots, n-1, \\
& a_{i} \leq \lambda_{i} \leq b_{i}, \quad \forall i=1, \ldots, n,
\end{aligned}
$$

where $c^{T}(x)=\left(d_{(1)}(x), \ldots, d_{(n)}(x)\right)$ and $h^{T}(y)=\left(d_{(1)}(y), \ldots, d_{(n)}(y)\right)$. (Recall that the optimal solution $\lambda^{*}$ satisfies $\lambda_{i}^{*} \in A B$ for $\left.i=1, \ldots, n\right)$.

Defining $\mu_{i}=\lambda_{i}-a_{i}, \beta_{i}=b_{i}-a_{i}$, for $i=1, \ldots, n, \alpha_{n}=0$, and $\alpha_{i}=a_{i+1}-a_{i}$ for $i=1, \ldots, n-1$, the formulation above reduces to:

$$
\begin{aligned}
\sum_{i=1}^{n} a_{i}\left(d_{(i)}(x)-d_{(i)}(y)\right)+\max & c^{T}(x) \mu-h^{T}(y) \mu \\
\text { s.t. } & \mu_{i}-\mu_{i+1} \leq \alpha_{i}, \quad \forall i=1, \ldots, n-1, \\
& \mu_{i} \leq \beta_{i}, \quad \forall i=1, \ldots, n, \\
& \mu_{i} \geq 0, \quad \forall i=1, \ldots, n .
\end{aligned}
$$

Setting $u_{0}=u_{n}=0$, the formulation of its corresponding dual problem is:

$$
\begin{aligned}
& \sum_{i=1}^{n} a_{i}\left(d_{(i)}(x)-d_{(i)}(y)\right)+ \min \sum_{i=1}^{n} \alpha_{i} u_{i}+\sum_{i=1}^{n} \beta_{i} t_{i} \\
& \text { s.t. } u_{i}-u_{i-1}+t_{i} \geq d_{(i)}(x)-d_{(i)}(y), \quad \forall i=1, \ldots, n, \\
& u_{i}, t_{i} \geq 0, \quad i=1, \ldots, n .
\end{aligned}
$$

Notice that the matrix defining the above linear program is totally unimodular since it is a flow matrix augmented by the identity matrix.

The above model corresponds to the following single commodity min-cost flow problem. (See Figure 2) For each $i=1, \ldots, n-1, u_{i}$ is the flow on the arc $(i+1, i)$, and for each $i=1, \ldots, n, t_{i}$ is the flow on left-arc $(0, i)$. For $i=1, \ldots, n$, the demand at node $i$ is $d_{(i)}(x)-d_{(i)}(y)$. Note that the demand can be of any sign. To account for the sign of the demand for $i=1, \ldots, n$, define $\delta_{i}^{+}(x)=\max \left\{0, d_{(i)}(x)-d_{(i)}(y)\right\}$ and $\delta_{i}^{-}(x)=\max \left\{0,-\left(d_{(i)}(x)-d_{(i)}(y)\right)\right\}$. The label attached to each edge in the graph has two coordinates. The left coordinate is the capacity upper bound on the flow, and the right one is the per unit cost of flow on the edge. The min-cost flow problem is to find the minimum cost of transporting $\sum_{i=1}^{n} \delta_{i}^{+}(x)$ units from the source node 0 to the destination $n+1$.

The fastest known strongly polynomial algorithm to solve a single commodity min-cost flow problem on a network with $n$ nodes and $m$ edges is $O(m S(n, m) \log n)$, where $S(n, m)$ is the time to solve the single source shortest path problem on a network with $n$ nodes and $m$ edges, having nonnegative lengths. (See Ahuja et al. 11.) This algorithm has $O(m \log n)$ scaling phases, where in each phase a shortest path problem is solved in $S(n, m)$ time. For a 
general graph $S(n, m)=O(m+n \log n)$ time. In our case $m$, the number of edges, satisfies $m \leq 4 n$, and $S(n, m)=O(n \log n)$. Moreover, the above flow problem is defined on a special planar network. It is a 3 -tree, i.e., its tree-width is bounded by 3 . The latter bound follows from the fact that this network has a separator consisting of the three nodes, $\{0,\lfloor n / 2\rfloor, n+1\}$. (Each of the two connected components obtained by removing these three nodes is a path consisting of at most $n / 2$ nodes. In particular, each component has a separator consisting of its median node).

Using the linear time algorithm in Henzinger et al., [10, designed for general planar graphs, or the more special algorithm for graphs with bounded treewidth in Chaudhuri and Zaroliagis, [6], in our case we have $S(n, m)=O(n)$. We conclude that computing $R_{y}(x)$ for a given $x$ and $y \in E Q$ can be done in $O\left(n^{2} \log n\right)$ time. Moreover, applying the results in [6], $R_{y}(x)$ can also be computed in $O\left(n \log ^{2} n\right)$ parallel time with $O(n / \log n)$ processors. With the above tools we can then directly use the parametric approach in Megiddo 13 with the modification in Cole [7, to obtain the next result.

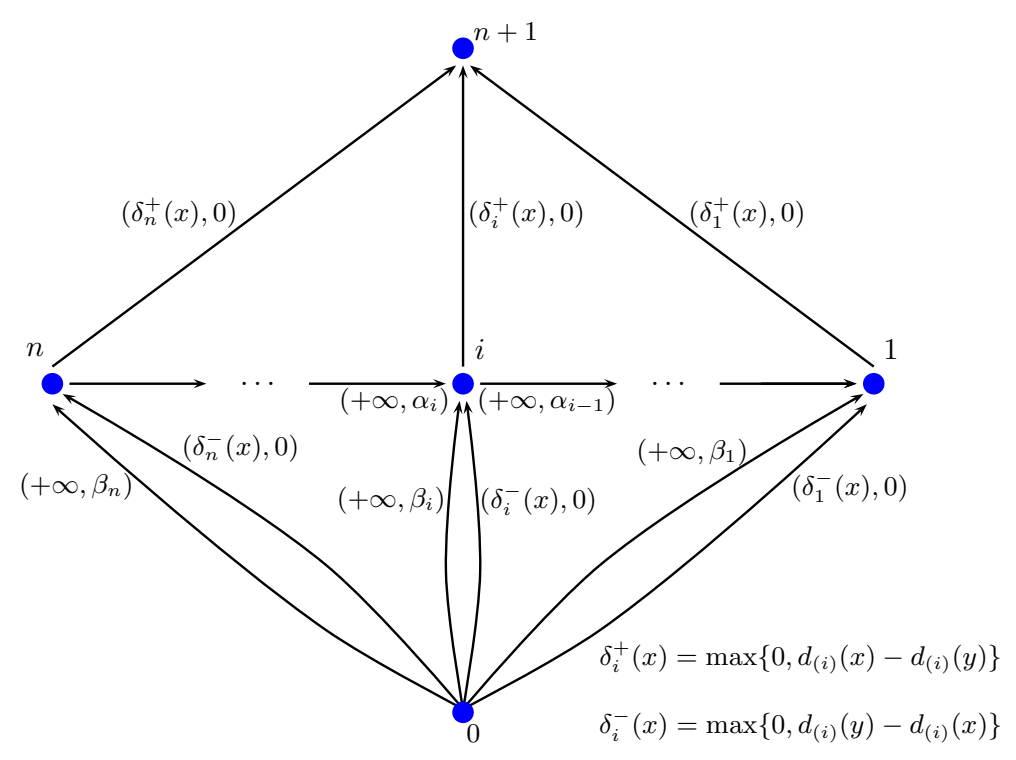

Fig. 2. Illustration of a $n$-diamond type network

Theorem 4. The total time to solve the single facility minimax regret convex ordered median problem on a general graph is $O\left(m^{2} n^{6} \log ^{4} n\right)$.

Finally, we use global convexity properties of the function $R(x)$ on tree graphs to solve the minimax regret convex ordered median problem on such graphs more efficiently. 
Theorem 5. The total time to solve the single facility minimax regret convex ordered median problem on a tree graph is $O\left(n^{6} \log ^{4} n\right)$. If the node weights are identical the total time reduces to $O\left(n^{5} \log ^{4} n\right)$.

\section{References}

1. Ahuja, R.K., Magnanti, T.L., Orlin, J.B.: Network Flows. Prentice Hall, New Jersey (1993)

2. Aissi, H., Bazgan, C., Vanderpooten, D.: Approximation of min-max and minmax regret versions of some combinatorial optimization problems. Eur. J. Oper. Res. 179, 281-290 (2007)

3. Averbakh, I.: On the complexity of a class of combinatorial optimization problems with uncertainty. Math. Program. 90, 263-272 (2001)

4. Averbakh, I., Berman, O.: An improved algorithm for the minmax regret median problem on a tree. Networks 41(2), 97-103 (2003)

5. Averbakh, I., Berman, O.: On the complexity of minmax regret linear programming. Eur. J. Oper. Res. 160, 227-231 (2005)

6. Chaudhuri, S., Zaroliagis, C.: Shortest paths in digraphs of small treewidth. Part I: Sequential algorithms. Algorithmica. 27, 212-226

7. Cole, R.: Slowing down sorting networks to obtain faster sorting algorithms. J. Assoc. Comput. Mach. 34, 200-208 (1987)

8. Conde, E.: An improved algorithm for selecting $p$ items with uncertain returns according to the minmax-regret criterion. Math. Program 100, 345-353 (2004)

9. Francis, R.L., Lowe, T.J., Tamir, A.: Aggregation error bounds for a class of location models. Oper. Res. 48, 294-307 (2000)

10. Henzinger, M.R., Klein, P., Rao, S., Subramanian, S.: Faster shortest-path algorithms for planar graphs. Journal of Computers and System Sciences 55, 3-23 (1997)

11. Hershberger, J., Suri, S.: Off-line maintenance of planar configurations. J. Algorithms 21(3), 453-475 (1996)

12. Kouvelis, P., Yu, G.: Robust discrete optimization and its applications. Kluwer Academic Publishers, Dordrecht (1997)

13. Megiddo, N.: Applying parallel computation algorithms in the design of serial algorithms. J. Assoc. Comput. Mach. 30, 852-865 (1983)

14. Megiddo, N.: Linear-time algorithms for linear programming in $\mathbb{R}^{3}$ and related problems. SIAM J. Comput. 12, 759-776 (1983)

15. Nickel, S., Puerto, J.: Location Theory: A unified approach. Springer, Heidelberg (2005)

16. Puerto, J., Rodriguez-Chia, A.M., Tamir, A.: Minimax regret single facility ordered median location problems on networks. Preprint Facultad de Matemáticas, Universidad de Sevilla (2007)

17. Ravi, R., Sinha, A.: Hedging uncertainty: approximation algorithms for stochastic optimization problems. Math. Program. 108, 97-114 (2006)

18. Tamir, A.: On the solution value of the continuous p-center location problem on a graph. Math. Oper. Res. 12, 340-349 (1987)

19. Tamir, A.: The $k$-centrum multi-facility location problem. Discrete Appl. Math. 109, 292-307 (2000) 